\title{
Raves y consumo de drogas desde una perspectiva epidemiológica y psicosocial: una revisión bibliográfica sistemática
}

\section{Raves and drug use from an epidemiologic and psychosocial approach: a bibliographic systematic review}

Fermín Fernández-CaLderón* ${ }^{\star * *}$; ÓsCar M. LOZANO-

ROJAs**; ANTONIO J. ROJAS-TEJadA ${ }^{\star \star *}$
* Jefatura de Inclusión Social y Gestión del Conocimiento. Agencia de Servicios Sociales y Dependencia de Andalucía.

** Departamento de Psicología Clínica, Experimental y Social. Universidad de Huelva.

${ }^{\star * \star}$ Departamento de Psicología. Universidad de Almería.

Enviar correspondencia a:

Fermín Fernández Calderón

Correo electrónico: fermin.fernandez@juntadeandalucia.es / fermin.fernandez@dpces.uhu.es

Dirección Postal: Calle Cueva de Gato n 3, portal 1, local 2.

CP. 41020 , Sevilla

\section{Resumen}

El elevado consumo de drogas que se produce en las raves y las caracteristicas propias de estas fiestas las convierten en un contexto recreativo de alto riesgo para la salud de los participantes. El objetivo de esta revisión es establecer una categorización de las investigaciones sobre raves y consumo de drogas a partir de sus objetivos y principales resultados. Se discuten las necesidades de investigación/conocimiento detectadas como fruto de esta revisión.

Para ello, se realizó una revisión sistemática de la literatura científica a través de las bases de datos Medline, Psycinfo y Psicodoc. Una vez aplicados los criterios de inclusión se obtuvieron 36 artículos, clasificados en seis categorías.

Los resultados han mostrado que 23 estudios tienen por objetivo el análisis del perfil psicosocial, la prevalencia y los patrones de consumo de drogas. Nueve estudios se centran en el riesgo asociado al consumo de drogas y otros nueve en sus efectos.

Dado el elevado riesgo asociado a las raves y la escasez de estudios empíricos detectado, se señala la necesidad de realizar más estudios empíricos. Las evidencias que permitan guiar las estrategias de prevención y reducción de daños con ravers son insuficientes. Se detecta la necesidad de estudiar: el policonsumo, las estrategias de reducción de daños, los efectos positivos y motivaciones.

Palabras clave: Rave, consumo de drogas, revisión bibliográfica sistemática, riesgo, reducción de daños. recibido: Septiembre 2012 aceptado: Marzo 2013

\section{Abstract}

The high drug use that occurs at raves and the specific characteristics of these parties make them a high risk recreational context the health of participants. The aim of this paper is to establish a categorization of research on drug use and raves according to their objectives and main results. Knowledge and research needs identified as a result of this review are discussed.

To this end, a systematic review of scientific literature through Medline, Psycinfo and Psicodoc was conducted. After applying the inclusion criteria, 36 papers were obtained, classified into six categories.

The results show that 23 studies aim psychosocial profile analysis, and the prevalence and patterns of drug use. Nine studies focus on risks related to drug use, and nine in the drug effects.

Given the high risk associated with raves and the scarcity of empirical studies identified, the need to develop further empirical studies is addressed. There is still insufficient evidence to guide intervention strategies to prevent risks and harms among ravers. There is also a need to explore: polydrug use, harm reduction strategies, positive effects and motivations.

Key words: Rave, drug use, bibliographic systematic review, risk, harm reduction 
E la década de los 80 un grupo de jóvenes británicos participa del movimiento Acid House en la isla de Ibiza (España), exportándolo a Reino Unido (Collin, 2002). Comienzan entonces a organizarse fiestas de música electrónica que, ante la dimensión adquirida y su vinculación al consumo de 3-4 metilendioximetanfetamina (MDMA), son perseguidas y prohibidas. Ello hace que se trasladen a la clandestinidad, surgiendo asi las raves a finales de los 80 (Anderson y Kavanaugh, 2007; Reynolds, 1999). Desde entonces se han expandido a distintos países y continentes (Weir, 2000), convirtiéndose en un fenómeno global.

Las raves eran, en su origen, de naturaleza underground o alternativa, con un marcado componente subcultural. Surgen como un movimiento al margen de lo establecido y en cierta manera reivindicativo y contracultural, caracterizado por el hedonismo, la libertad y la colectividad (Anderson y Kavanaugh, 2007; Weir, 2000). La presión legal-policial y las implicaciones económicas de estas fiestas para promotores y organizadores favorecieron que, con el paso del tiempo, parte del movimiento se integrara en los circuitos comerciales de ocio. Ello dio lugar a las raves de tipo comercial, convencional o mainstream.

A pesar de que ambos tipos de raves comparten numerosos elementos e incluso participantes, existen diferencias. Las raves underground se caracterizan por: música electrónica y baile; son organizadas por los propios asistentes, sin fines comerciales; son alternativas a los circuitos recreativos convencionales y de larga duración; se celebran en lugares ocultos o de difícil acceso; y en ellas se da un elevado consumo de drogas (Anderson y Kavanaugh, 2007; Boeri, Sterk y Elifson, 2004; Gross, Barrett, Shestowsky y Pihl, 2002). Las raves mainstream se diferencian de las anteriores por su carácter comercial (económico y promocional), anunciándose públicamente, y celebrándose en clubs, discotecas o locales alquilados (Boeri et al, 2004).

Durante la década de 90, las fiestas raves acaparan el interés de la comunidad científica por dos aspectos fundamentalmente. Por un lado, desde disciplinas como la Sociología, se produce un interés por su entidad de subcultura (p.e. Bennett, 1999, 2001; Hill, 2002). Por otro, el consumo de drogas que se realiza en estos contextos ha motivado el interés de investigadores en el campo de las drogodependencias, especialmente por su vinculación al policonsumo (European Monitoring Centre for Drugs and Drug Addiction [EMCDDA], 2009). Estos dos aspectos han determinado que las investigaciones realizadas sobre raves hayan sido planteadas desde dos posiciones diferenciadas: una perspectiva cultural y otra epidemiológica o de la salud pública.

Los estudios culturales han entendido las raves como una subcultura, centrando su foco de interés en los significados asociados a ellas (empatía, conectividad, placer, libertad, etc.). Desde este posicionamiento, el consumo de drogas no es el eje central de las raves, sino un elemento más que interacciona con la música, el baile, el contacto entre los asistentes y el sentimiento de pertenencia grupal. Por su parte, desde la perspectiva epidemiológica o de la salud pública se ha focalizado en el riesgo para la salud asociado a las raves, centrándose en las consecuencias negativas del consumo de drogas y en la peligrosidad y el riesgo que implican.

Independientemente de la perspectiva que se adopte, las raves son consideradas como el contexto recreativo donde el uso de drogas puede suponer un mayor riesgo para la salud de los participantes. Por todo ello, y teniendo en cuenta la gran dimensión adquirida desde sus inicios, se trata de un entorno susceptible de actuaciones preventivas y de reducción de riesgos.

Para ser eficaces, dichas actuaciones deben estar fundamentadas empíricamente. En este sentido, una revisión sistemática puede aportar beneficios en un doble sentido. Por una parte, sintetizando el conocimiento que existe sobre el fenómeno rave y el consumo de drogas, de manera que permita guiar el diseño de estrategias preventivas y la toma de decisiones en este ámbito. Por otra, señalando necesidades de investigación, de manera que futuros estudios aborden los aspectos susceptibles de un mayor conocimiento.

Así, el objetivo de esta revisión es establecer una categorización de las investigaciones sobre rave y consumo de drogas a partir de sus objetivos y principales resultados. Para ello se revisó la literatura científica. Se discuten las necesidades de investigación/conocimiento detectadas como fruto de esta revisión.

\section{Método}

\section{Estrategia de búsqueda y criterios de selección}

Se realizó una búsqueda bibliográfica para identificar estudios relacionados con el consumo de drogas en fiestas rave. No se establecieron restricciones temporales, finalizando la búsqueda el 31 de julio de 2012. Las bases de datos consultadas fueron: Medline, Psycinfo y Psicodoc. La elección de estas bases de datos se realizó considerando la propia naturaleza de las raves. Se trata de un fenómeno asociado al consumo de drogas donde están presentes aspectos psicológicos y/o culturales (Psyinfo y Psicodoc) y aspectos directamente relacionados con la salud (Medline). Igualmente, la fuerte vinculación contextual de las raves llevó a los investigadores a consultar Psicodoc, que aúna trabajos del ámbito español y de América Latina.

Como términos de búsqueda se introdujeron los términos "rave*" y "drug*", acotando la búsqueda a los campos "títuIo" o "resumen" de los artículos. Además, a través de diferentes búsquedas, estos términos fueron introducidos de forma combinada con los siguientes: club / party / dance / electronic / music / consume / use / pattern / polydrug / polysubstance / recreational / young / consumption. En este caso, los términos "rave" y "drug" se introducían como obligatorios en los campos "título" o "resumen". Para el resto de términos se establecía la condición de que al menos uno de ellos apareciera en alguno de los campos del artículo completo. Además, se realizaron también búsquedas en las que una combinación de términos debía aparecer en algún campo del documento. 
Para identificar artículos en español, estos términos fueron también introducidos en dicho idioma (excepto rave, cuya traducción es similar). Además, para detectar otros estudios no identificados en la búsqueda se examinaron los listados de referencias de los artículos seleccionados.

La búsqueda finalizó una vez que la combinación de los términos anteriores mostraba duplicidades, resultando un total de 242 artículos publicados. Inicialmente, dos investigadores analizaron de forma independiente los títulos y abstracts, discutiendo los desacuerdos encontrados y seleccionando exclusivamente aquellos relacionados con raves $(n=74)$. A continuación, ambos autores procedieron al análisis de los artículos completos mediante su lectura, comprobando que cumplían los criterios de inclusión fijados. Estos fueron los siguientes:

1. Tener como objetivo principal de estudio variables o aspectos de las raves. Se pretendía evitar la revisión de artículos que incluyeran asistentes a raves por cumplir alguna característica de interés para el objetivo de su estudio. Por ejemplo, es muy común la inclusión de ravers en artículos dedicados al estudio de los efectos de la MDMA, siendo el objetivo de estudio la MDMA y no el consumo que hacen los ravers de esta droga.

2. Reflejar la asistencia a rave o el hecho de que la muestra haya sido captada en estas fiestas. El total de la muestra, y no sólo una parte, debe haber sido captada entre usuarios de raves. Con ello se pretendía incluir artículos cuyos resultados fueran atribuibles exclusivamente a los ravers.

3. Debe tratarse, bien de estudios empíricos con datos originales, o bien de revisiones sobre el fenómeno rave. No se incluyeron trabajos teóricos.

4. Las revisiones deben serlo sobre el fenómeno rave y el consumo de drogas, haciéndose explícito en el título y/o objetivo de las mismas.

Tras analizar los 74 artículos previamente seleccionados y comprobar si cumplían con los criterios prefijados, los dos investigadores acordaron considerar aptos para esta revisión 36 artículos.

\section{Categorización de estudios según objetivos y resultados}

Se llevó a cabo un proceso de categorización de los artículos seleccionados atendiendo al objetivo del estudio revisado y los principales resultados mostrados. Para ello, se revisaron los objetivos de los artículos seleccionados, consensuando el nombre y definición operativa de cada categoría, estableciéndose seis. Para la adscripción de los artículos a cada categoría, dos investigadores lo hicieron de forma independiente. Aquellos artículos en que los investigadores habían mostrado desacuerdo fueron discutidos hasta llegar a un consenso. Las categorías establecidas, su título y definición fueron las siguientes:

- Categoría 1: Perfil psicosocial, prevalencia y patrones de consumo. Artículos que tienen por objetivo describir el perfil psicosocial de los ravers, la prevalencia de consumo de una o diferentes drogas y/o los patrones de consumo.
- Categoría 2: Riesgo. Artículos que tienen por objetivo analizar aspectos relacionados con el riesgo de consumir drogas en raves. Pueden diferenciarse aquí dos tipos de estudios: Aquellos que tienen por objetivo conductas 0 comportamientos (conducción, relaciones sexuales, compra de drogas, conductas de prevención); y aquellos que estudian variables de tipo interno o no observables (percepción de riesgo, relación entre riesgo y experiencia en el consumo, riesgo de desarrollar dependencia).

- Categoría 3: Efectos y motivación. Artículos que se interesan por los efectos percibidos y/o esperados, así como por la motivación en relación al consumo. Estos dos elementos han sido incluidos en una sola categoría por dos razones. Por un lado, una de las razones o motivaciones para consumir una droga es precisamente el efecto que provoca. Por otro, en muchos de los estudios revisados, motivación y efectos están explícitamente identificados. Dentro de esta categoría los temas explorados han sido: efectos negativos, efectos positivos, motivaciones o razones para usar drogas.

- Categoría 4: Aspectos culturales. Artículos que tienen por objetivo principal el estudio de alguno de los aspectos culturales de las raves, en concreto: solidaridad, naturaleza de la experiencia, fragmentación, identidad, valores. Los trabajos en que la temática cultural es abordada de forma secundaria no han sido incluidos en esta categoría.

- Categoría 5: Revisiones. Artículos cuyo objetivo ha sido revisar diferentes aspectos de las fiestas rave y el consumo de drogas, y así lo han explicitado en el título y objetivo.

- Categoría 6: Otros objetivos. En esta categoría se han incluido estudios cuyos objetivos no se corresponden con las categorías anteriores.

\section{Resultados}

\section{Descripción de estudios}

Del total de estudios revisados, 36 cumplen con los criterios de inclusión fijados. En la tabla 1 se muestran los artículos detectados así como el país donde han sido realizados, el tipo de rave que estudian, el tamaño muestral, los principales objetivos y la/s categoria/s en que han sido identificados.

La mayor parte de la investigación sobre rave se ha desarrollado en EEUU (13 artículos). En Reino Unido, al igual que en Paises Bajos han sido publicados seis artículos, mientras que Australia, Canadá y España cuentan con tres publicaciones.

Respecto al tipo de fiesta, lo más habitual es encontrar trabajos empíricos que han accedido a raves convencionales. Los trabajos de Boys, Lenton y Norcross. (1997), Lenton, Boys y Norcross (1997) y Lenton y Davidson (1999) informan de que entre sus participantes había asistentes tanto a raves underground como mainstream. Forsyth (1996) también accede a una muestra con ambos tipos de ravers. Los únicos estudios con muestras underground son, por un lado, el de Riley, Morey y Griffin (2008) que realiza un análisis cualitativo con 19 ravers underground que consumen ketamina. Por otro, los 
trabajos de Fernández-Calderón et al. (2011a, 2011b, 2012), que acceden a 248 ravers underground.

El tamaño de las muestras puede considerarse, en la mayoría de los casos, reducido. En los casos donde se aprecia un alto número de participantes se trata de raves comerciales (p.e. Krul y Girbes, 2011a; Krul, Blankers y Girbes, 2011b).

Tabla 1

Descripción de estudios sobre rave detectados tras la revisión

\section{Categorización según objetivos y resultados}

Categoría 1. Perfil psicosocial de los asistentes, prevalencia y patrones de consumo

La mayoría de estudios revisados (23 de 36) han sido adscritos a esta categoría. Se trata, en general, de jóvenesadultos, la mayoría con edades comprendidas entre los

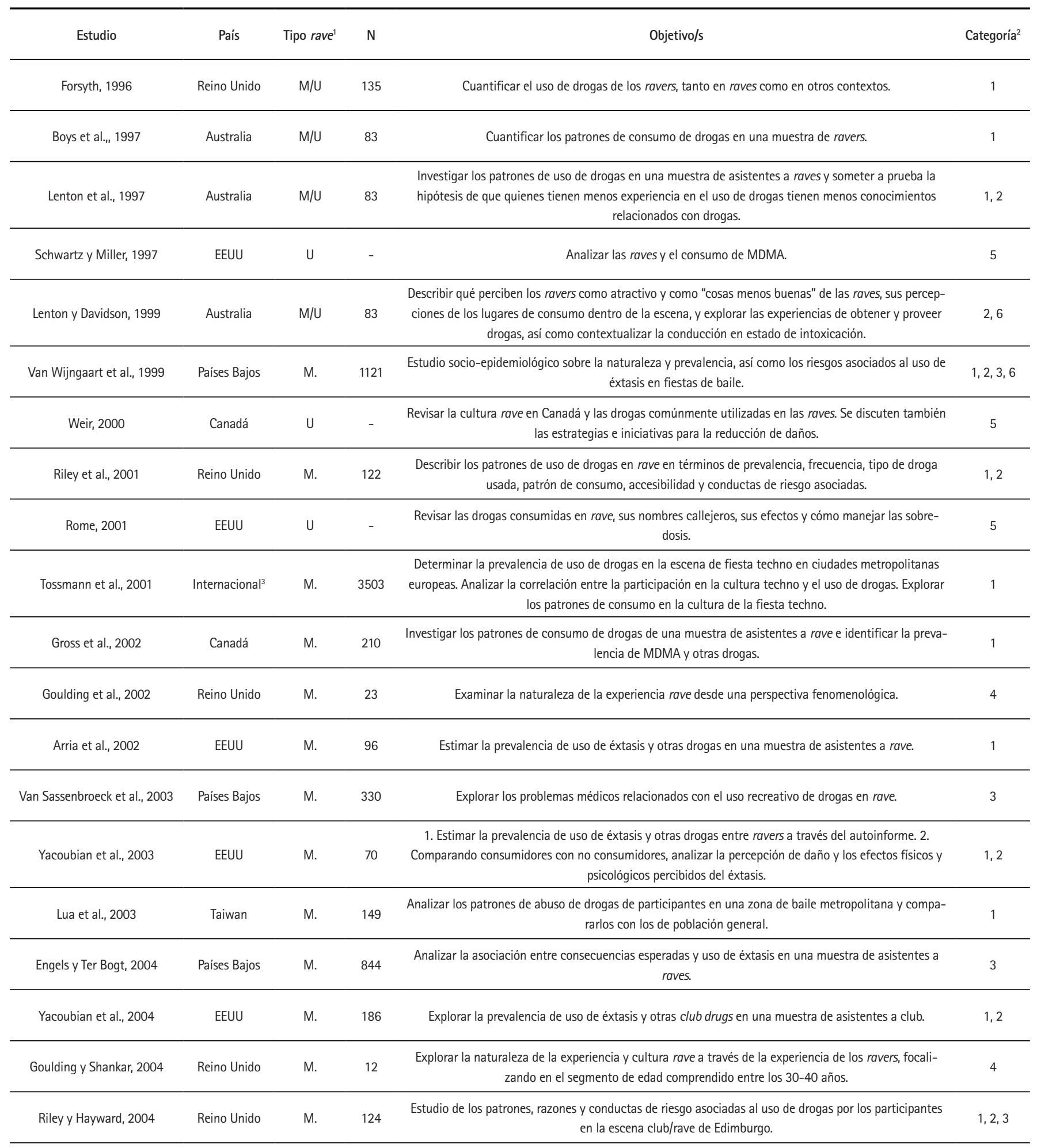




\begin{tabular}{|c|c|c|c|c|c|}
\hline Ter Bogt y Engels, 2005 & Paises Bajos & M. & 490 & $\begin{array}{l}\text { Describir los patrones de uso de MDMA en diferentes tipos de rave, para identificar un set de motivos } \\
\text { para su uso y analizar si el tipo de fiesta y las motivaciones están asociadas con los efectos positivos y } \\
\text { negativos percibidos de la MDMA. }\end{array}$ & 1,3 \\
\hline Barrett et al., 2005 & Canadá & $S / E$ & 186 & $\begin{array}{l}\text { Describir los patrones de policonsumo simultáneo caracteristicos de las raves, documentando la canti- } \\
\text { dad, orden y tipo de todas las sustancias consumidas en una sola ocasión a través de una muestra de } \\
\text { asistentes a rave. }\end{array}$ & 1 \\
\hline McCaughan et al., 2005 & EEUU & $\mathrm{S} / \mathrm{E}$ & 36 & Presentar una tipología epidemiológica preliminar de jóvenes que asisten a rave. & 1 \\
\hline Yacoubian y Wish, 2006 & EEUU & M. & 96 & Explorar la validez del autoinforme sobre el uso de MDMA entre asistentes a rave. & 6 \\
\hline Anderson y Kavanaugh, 2007 & EEUU & $U$ & - & $\begin{array}{c}\text { Revisión que compara la situación actual de las raves con las de los } 90 \text {. Se discute cómo los estudios } \\
\text { culturales y de la salud pública definen las raves y las han estudiado. }\end{array}$ & 5 \\
\hline Yacoubian y Peters, 2007 & EEUU & M. & 283 & $\begin{array}{l}\text { Explorar la asociación entre el uso de éxtasis y otras club drugs, y las conductas sexuales de alto riesgo } \\
\text { en una muestra de } 283 \text { asistentes a rave. }\end{array}$ & 1,2 \\
\hline Kavanaugh y Anderson, 2008. & EEUU & M. & 49 & Analizar la relación entre solidaridad y uso de drogas en fiestas de música electrónica. & 4 \\
\hline Riley et al., 2008 & Reino Unido & U. & 19 & $\begin{array}{l}\text { Examinar el rol de las drogas en la identidad subcultural y las prácticas de consumo, a través del análisis } \\
\text { de las múltiples y potencialmente contradictorias interpretaciones de que se sirven los participantes en } \\
\qquad \text { raves underground cuando hablan de ketamina. }\end{array}$ & 4 \\
\hline Moloney et al., 2008 & EEUU & M. & 206 & $\begin{array}{l}\text { Analizar la relación entre el uso de drogas y la identidad étnica en las narrativas de } 206 \text { jóvenes asio- } \\
\qquad \text { americanos de la escena de baile club-rave. }\end{array}$ & 4 \\
\hline Hunt et al., 2009a & EEUU & $S / E$ & 300 & $\begin{array}{l}\text { Analizar las diferentes combinaciones de uso de drogas utilizadas por un grupo de jóvenes que atienden } \\
\text { a raves, clubs y fiestas de baile (policonsumo) en San Francisco. Se exploran también las razones por las } \\
\text { que unas combinaciones son realizadas y otras evitadas. }\end{array}$ & $1,2,3$ \\
\hline $\begin{array}{l}\text { Fernández-Calderón et al., } \\
\qquad 2011 \text { a }\end{array}$ & España & U. & 248 & $\begin{array}{l}\text { 1. Analizar el perfil sociodemográfico de jóvenes asistentes a fiestas raves underground. 2. Aportar } \\
\text { prevalencias de consumo de drogas en fiestas rave underground realizadas en España. 3. Analizar los } \\
\text { patrones de policonsumo de los asistentes a estas fiestas. 4. Establecer clústers de consumidores según } \\
\text { el patrón de consumo. }\end{array}$ & 1 \\
\hline $\begin{array}{l}\text { Fernández-Calderón et al., } \\
\text { 2011b }\end{array}$ & España & U. & 248 & $\begin{array}{c}\text { Analizar las diferencias en el patrón de consumo y el perfil sociodemográfico de hombres y mujeres que } \\
\text { asisten a fiestas rave }\end{array}$ & 1 \\
\hline Krul y Girbes, 2011a & Países Bajos & M. & 22604 & $\begin{array}{l}\text { Determinar las alteraciones de salud y medir la severidad de los incidentes informados durante una } \\
\text { experiencia de } 9 \text { años de atenciones de primeros auxilios relacionados con el GHB en las instalaciones } \\
\text { de primeros auxilios situadas en fiestas rave. }\end{array}$ & 3 \\
\hline Krul et al., 2011b & Paises Bajos & M. & 27897 & $\begin{array}{l}\text { Describir la observación de } 12 \text { años de incidentes relacionados con drogas ocurridos en fiestas rave en } \\
\qquad \text { Holanda }\end{array}$ & 3 \\
\hline $\begin{array}{l}\text { Fernández-Calderón et al., } \\
\qquad 2012\end{array}$ & España & U. & 248 & $\begin{array}{l}\text { 1. Describir los efectos positivos y negativos percibidos por asistentes a fiestas rave underground. } 2 \text {. } \\
\text { analizar las relaciones existentes entre diferentes drogas y los efectos positivos y negativos. }\end{array}$ & 3 \\
\hline
\end{tabular}

${ }^{1}$ M.: mainstream/U.: underground /S.E.: sin especificar

${ }^{2}$ 1.: Perfil psicosocial, prevalencia y patrones de consumo. 2.: Riesgos. 3.: Efectos y motivaciones. 4.: Culturales. 5.: Revisión. 6.: Otros

${ }^{3}$ Holanda, Alemania, España, Respública Checa, Italia, Suiza, Austria.

21-23 años (Barrett, Gross, Garand y Pihl, 2005; Gross et al., 2002; Forsyth, 1996; Krul y Girbes, 2011a; Krul et al., 2011b; McCaughan, Carlson, Falck y Siegal, 2005; Riley y Hayward, 2004; Riley, James, Gregory, Dingle y Cadger, 2001; Ter Bogt y Engels, 2005; Tossmann, Boldt y Tensil, 2001; Yacoubian y Peters, 2007). También se encuentran jóvenes con edades inferiores a los 21 años (Arria, Yacoubian, Fost y Wish, 2002; Boys et al., 1997; Hunt, Evans, Moloney y Bailey, 2009a; Van Wijngaart et al., 1999; Yacoubian, Boyle, Harding y Loftus, 2003) y superiores a los 23 (Barrett et al., 2005; Boys et al., 1997; Fernández-Calderón et al., 2011a, 2011b, 2012; Forsyth, 1996; Riley et al., 2001; Van Wijngaart et al., 1999).

Hombres y mujeres participan en las raves casi en igual proporción, siendo el número de hombres ligeramente superior (Barrett et al., 2005; Boys et al., 1997; Fernández-Calderón et al., 2011a; Hunt et al., 2009a; Krul y Girbes, 2011a; Krul et al., 2011b; Riley y Hayward, 2004; Yacoubian et al., 2003; Yacoubian y Peters, 2007).

Los ravers son considerados como un grupo normalizado y/o socialmente integrado (Fernández-Calderón et al., 2011a; Goulding, Shankar y Elliott, 2002; Tossman et al., 2001). Una variable fundamental en este sentido es la formación académica. La revisión bibliográfica ha mostrado que se trata de jóvenes con un nivel académico acorde a su cohorte. Por ejemplo, Hunt et al. (2009a), en una muestra de 20 años de edad de media, encuentran que el 21,4\% había finalizado estudios universitarios. En la muestra de Barrett et al. (2005), el 59,5\% había finalizado estudios post-secundarios. Riley et al. (2001) acceden a una muestra de asistentes a raves en la que prácticamente el $40 \%$ estaba en ese momento realizando estudios 
Tabla 2

Prevalencias de consumo de drogas en estudios realizados en raves (\%)

\begin{tabular}{|c|c|c|c|c|c|c|c|c|c|c|c|c|}
\hline \multirow[b]{2}{*}{ Estudio $^{1}$} & \multicolumn{6}{|c|}{ ALGUNA VEZ EN LA VIDA } & \multicolumn{6}{|c|}{ ÚLTIMO AÑO } \\
\hline & Ext. ${ }^{2}$ & Anf. $^{2}$ & $\angle S D$ & Coc. $^{2}$ & Ket. $^{2}$ & $G H B$ & Ext. & Anf. & $\angle S D$ & Coc. & Ket. & $G H B$ \\
\hline Forsyth, 1996 & 91,1 & 93,3 & 91,9 & 70,4 & 14,8 & - & 87,4 & 77 & 78,5 & 58,5 & 7,1 & - \\
\hline Lenton et al., 1997 & 75,9 & 68,7 & 90,4 & 19,3 & 12 & - & - & - & - & - & - & - \\
\hline Wijngaart et al., 1999 & 81 & 63 & 26 & 40 & - & - & 76 & 50 & 16 & 34 & - & - \\
\hline Riley et al., 2001 & - & - & - & - & - & - & 82 & 81,1 & 30,3 & 38,5 & 12,3 & \\
\hline Gross et al., 2002 & 65,2 & 73,3 & 56,2 & 34,8 & 13,8 & 18,6 & - & - & - & - & - & - \\
\hline Arria et al., 2002 & 89 & 22 & - & 44 & - & - & 76 & - & - & 42 & - & - \\
\hline Yacoubian et al., 2003 & 86 & & & & & & & & & & & \\
\hline Yacoubian et al., 2004 & & & & & & & 10 & 3 & 4 & 9 & 2 & 1 \\
\hline Riley y Hayward, 2004 & - & - & - & - & - & - & 79,8 & 53,2 & 33,1 & 62,9 & 15,3 & - \\
\hline Yacoubian y Peters, 2007 & 71 & & & & 40 & 18 & 34 & & & & & \\
\hline Fernández-Calderón et al., 2011 & $98,8^{3}$ & 93,5 & 72,9 & 93,9 & 54,1 & 16,1 & - & - & - & - & - & - \\
\hline
\end{tabular}

1 Los datos sobre prevalencia del estudio internacional realizado por Tossmann et al. (2001) no han sido incluidos por no ofrecer las prevalencias de las siete ciudades participantes de manera conjunta.

${ }^{2}$ Ext.: éxtasis/Anf:: anfetamina / Coc.: cocaina / Ket.: ketamina

${ }^{3}$ Este estudio diferencia entre éxtasis en polvo y en pastilla. Los datos que se muestran aqui corresponden a su presentación en polvo.

Tabla 3.

Policonsumo de drogas en raves (\%)

\begin{tabular}{cccc}
\hline Drogas & $\begin{array}{c}\text { Boys et al., } \\
1997\end{array}$ & $\begin{array}{c}\text { Barrett et al., } \\
2005\end{array}$ & $\begin{array}{c}\text { Fernández-Calderón et al., } \\
\text { 2011a }\end{array}$ \\
\hline Cannabis & 51,8 & 64,9 & 76,7 \\
Alcohol & 18,1 & 52,2 & 94,4 \\
Éxtasis & 27,7 & 50 & $57,3^{*}$ \\
Anfetamina & 34,9 & 47,8 & 74,7 \\
Ketamina & - & 8,6 & 23,8 \\
Tranquilizantes & 6 & - & 2 \\
GHB & - & 7 & 0,04 \\
Cocaína & - & 9,1 & 28,2 \\
LSD & 34,9 & $<5$ & 22,9 \\
\hline
\end{tabular}

${ }^{*}$ Este estudio diferencia entre éxtasis en polvo y en pastilla. Los datos que se muestra aqui corresponden a su presentación en polvo.

universitarios (edad media, 21,6 años). En España, Fernández-Calderón et al. (2011a), con una muestra de 23,9 años de media de edad, encuentran que el 24,3\% había finalizado estudios universitarios.

Las prevalencias de consumo "alguna vez en la vida" y "en el último año" son elevadas (tabla 2), superando las detectadas en población general (EMCDDA, 2011; Observatorio Español sobre Drogas, 2007) u otros consumidores recreativos (Adlaf y Smart, 1997; Barrett, Darredeu y Pihl, 2006).

Las prevalencias más elevadas se encuentran para el consumo de éxtasis, anfetaminas y LSD. La cocaína presenta también elevadas prevalencias de consumo. En el indicador de consumo en el último año, sin considerar el estudio de Yacoubian et al., (2004) (por presentar prevalencias excepcionalmente bajas), las prevalencias de consumo de éxtasis varian en

un rango de $34 \%-87,4 \%$. El consumo de anfetaminas lo hace entre 50\%-81,1\% y el de LSD entre 16\%-78\%.

Las raves son un fenómeno global, pero ello no significa que sea homogéneo, especialmente en lo que a patrones de consumo de drogas se refiere. Por ejemplo, Tossmann et al. (2001) realizaron un estudio multicéntrico en raves de siete ciudades europeas. Sus resultados muestran notables diferencias entre las ciudades respecto al consumo de cocaína, opiáceos y éxtasis. El estudio de Fernández-Calderón et al. (2011), con asistentes a fiestas rave underground, muestra dos perfiles de consumidores en sus resultados: uno con una tendencia a consumir estimulantes y otro que también consume estimulantes pero se inclina en mayor medida por los alucinógenos.

El estudio del policonsumo de drogas, a pesar de tratarse de una conducta normativa en rave, ha sido escaso. Sólo cuatro de los 36 estudios analizados se han planteado el policonsumo como objetivo principal (Barrett et al., 2005; Boys et al., 1997; Fernández-Calderón et al., 2011a; Hunt et al., 2009a), mientras que en otros casos ha sido abordado de manera transversal (p.e. Fernández-Calderón et al., 2012; Gross et al., 2002; Riley et al., 2001; Riley y Hayward, 2004).

De los cuatro estudios que analizan el policonsumo en raves, uno de ellos (Hunt et al., 2009) utiliza metodología cualitativa. Los otros tres, lo hacen cuantitativamente, recogiendo información sobre el consumo de drogas asociado a la "última rave" a la que acudieron los participantes (tabla 3 ). Sus resultados muestran que las drogas más vinculadas a las raves fueron: cannabis, alcohol, anfetaminas y MDMA. Además, aportan información sobre el número de drogas consumidas en la última rave. En el estudio de Boys et al. (1997), de 18 posibles sustancias, el 67,5\% de los asistentes había consumido dos 0 más (sin incluir tabaco). En el estudio de Barrett et al. (2005), 
sin incluir tabaco, la media de drogas consumidas fue de 2,5 (de 21 posibles sustancias). Fernández-Calderón et al. (2011a) detectan una media de 4,9 de 15 posibles (incluyendo tabaco). En este último estudio, el $42,8 \%$ de los participantes había consumido cinco o seis drogas en la última rave, mientras que el 18,9\% consumió siete 0 más.

Dos de estos estudios (Boys et al., 1997; Fernández-Calderón et al., 2011a) analizan, además, el consumo antes, durante y después de la rave, detectando un mayor uso de sustancias depresoras después de la fiesta.

Hunt et al. (2009a), utilizando metodología cualitativa, analizan los patrones de policonsumo y las razones para elegir unas combinaciones y evitar otras. Además, profundizan en las estrategias de los consumidores para aumentar los efectos positivos y evitar los negativos cuando llevan a cabo conductas de policonsumo. Sus resultados apuntan hacia estas personas como activas en el policonsumo de drogas respecto a la administración de placeres y evitación de riesgos.

\section{Categoría 2. Riesgos derivados del consumo}

En esta categoria se han incluido nueve estudios que analizan diferentes aspectos relacionados con el riesgo de consumir drogas en raves (tabla 1), los cuales han sido diferenciados según estudien comportamientos o variables de tipo interno.

Entre los estudios que tienen por objetivo comportamientos, tres de ellos (Lenton y Davidson, 1999; Riley et al., 2001; Riley y Hayward, 2004) analizan la compra de drogas y la conducción bajo los efectos de las drogas como factores de riesgo. Sus resultados señalan, por una parte, que los ravers prefieren comprar las drogas a fuentes fiables y con anterioridad a la rave. Por otra, que los ravers asumen riesgos asociados a la conducción bajo los efectos de las drogas. Otros estudios (Riley et al., 2001, Riley y Hayward, 2004; Yacoubian y Peters, 2007) han mostrado que los ravers asumen un elevado riesgo cuando mantienen relaciones sexuales bajo los efectos de las drogas.

Respecto a los estudios que analizan variables de tipo interno o no observable, Lenton et al. (1997) y Van Wijngaart et al. (1999), concluyen que la experiencia en el consumo (en términos de duración del consumo en el tiempo) se relaciona con una menor probabilidad de riesgos. Yacoubian et al. (2004) y Yacoubian y Peters (2007) estudian el riesgo de desarrollar dependencia a éxtasis entre los ravers, poniendo de manifiesto el escaso riesgo de desarrollar este trastorno entre sus participantes. Yacoubian et al. (2003) analizan la percepción de riesgo entre consumidores y no consumidores de éxtasis. Sus resultados indican que estos últimos perciben mayores consecuencias negativas físicas y psicológicas asociadas al consumo de éxtasis.

Categoría 3. Efectos percibidos y las motivaciones para el consumo

En esta categoría han sido identificados 9 trabajos (ver tabla 1). La información aportada por los diferentes estudios muestran que éstos se han centrado en: efectos negativos percibidos de las drogas (Fernández-Calderón et al., 2012; Hunt et al., 2009a; Krul y Girbes, 2011a; Krul et al., 2011b; Riley y Hayward, 2004; Ter Bogt y Engels, 2005; Van Sassenbroeck et al., 2003; Van Wijngaart et al., 1999), los efectos positivos percibidos de las drogas (Hunt et al., 2009a; Fernández-Calderón et al., 2012; Ter Bogt y Engels, 2005), las motivaciones o razones para usar drogas (Engels y Ter Bogt, 2004; Riley y Hayward, 2004, Ter Bogt y Engels, 2005; Van Wijngaart et al., 1999).

Los efectos negativos de las drogas han sido el tópico más estudiado dentro de esta categoría. Ocho de los nueve estudios revisados aquí analizan este tipo de información, recogiendo la prevalencia de efectos negativos como: náusea, insomnio, falta de concentración, pérdida de apetito, dolor de cabeza, etc. Por ejemplo, Fernández-Calderón et al. (2012) analizan los efectos negativos más percibidos asociados al consumo de drogas de los participantes en la última rave a la que acudieron, destacando: cansancio $(78,2 \%)$, bruxismo $(55,2 \%)$, falta de concentración $(54,4 \%)$ y pérdida de sueño $(53,6 \%)$. Además, analizan la contribución de las diferentes drogas a la percepción de los diferentes efectos, destacando por ejemplo, la contribución del consumo de ketamina a la percepción del efecto de "mal viaje", cuya probabilidad de ser percibido se ve aumentada casi seis veces entre quienes consumieron la droga.

La diferenciación de los efectos negativos y positivos en este apartado ha pretendido poner de manifiesto el mayor número de artículos dedicados a los primeros. Esta es una característica asociada no sólo a las raves, sino a los estudios sobre consumo de drogas en general. Hunt y Evans (2008) han criticado enfáticamente este hecho etiquetando el placer que aportan las drogas como "el gran inmencionable" y preguntándose "¿por qué tantos estudios sobre consumo de sustancias han ignorado esta característica fundamental?" (p.1).

Sólo tres de los 36 estudios revisados han profundizado y recogido los efectos positivos de las drogas en raves. Ter Bogt y Engels (2005) se centran sólo en la MDMA. Al igual que hicieran con los efectos negativos, relacionan los efectos positivos con las motivaciones hacia el consumo, llegando a la conclusión de que las principales razones para tomar MDMA son: energía, euforia y facilitación de las relaciones sociales. El estudio de Fernández-Calderón et al. (2012) recoge 11 efectos positivos asociados al consumo de diferentes drogas en la última rave, coincidiendo con Ter Bogt y Engels (2005) en que el más percibido fue "euforia, bienestar, felicidad" (90,3\%). Otros efectos percibidos en gran proporción por los ravers fueron: conexión con la música $(75,4 \%)$, potenciación de los sentidos $(66,5 \%)$ y aumento/mejora de la comunicación con los demás $(65,3 \%)$. Al igual que hicieran con los efectos negativos, los autores analizan la contribución de las diferentes drogas a la aparición de los efectos, detectando que la MDMA incrementa significativamente la probabilidad de aparición de gran parte de los efectos positivos recogidos. Hunt et al. (2009a) analizan los efectos positivos de distintas combinaciones de drogas.

Entre los trabajos que analizan la motivación para consumir drogas en las raves, Riley y Hayward (2004) destacan: relajarse $(68,5 \%)$, aumento de la confianza $(64,8 \%)$ y energía para el baile (55,6\%). Ter Bogt y Engels (2005) relacionan la motivación hacia el consumo con los efectos negativos, destacando la relación entre la motivación de consumir por integrarse con los iguales y la mayor probabilidad de sufrir efectos negativos. Engels y Ter Bogt (2004) tratan de obtener conoci- 
miento relacionado con la forma en que las expectativas sobre los efectos del éxtasis afectan a la decisión de consumirlo o no, concluyendo que cuando los efectos esperados (físicos y emocionales) son percibidos como negativos existe mayor probabilidad de no consumir y viceversa.

\section{Categoría 4. Según el estudio de elementos culturales}

Una cuarta categoría aglutina siete estudios que tienen por objetivo principal elementos puramente culturales (identidad, cultura, comunidad, solidaridad, significados...). Sus aportaciones permiten profundizar en aquellos valores y elementos que han aportado (y aportan) al movimiento rave esa entidad de "subcultura" compartida por muchos usuarios e investigadores. Estos trabajos han realizado un análisis cualitativo de la información.

Kavanaugh y Anderson (2008) exploran la relación entre solidaridad y rave, concluyendo que el uso de drogas contribuye a la solidaridad en los eventos de música y baile. Además, diferencian dos tipos de solidaridad que se ven influenciadas por el consumo de drogas, la afectivo-social y la conductualorganizacional.

Goulding et al. (2002) examinan la naturaleza de la experiencia rave en términos de identidad y fragmentación, apuntando hacia los ravers como personas normalizadas y socialmente integradas que fragmentan su identidad sumergiéndose en la cultura rave los fines de semana. Sus resultados sugieren que la experiencia rave está muy unida a los conceptos de identidad, escape, alienación y prolongación del hedonismo. Esta misma autora, en el año 2004, examina la naturaleza y significados de la cultura rave en el segmento etario de 30-40 años a través personas que cataloga como "cognitivamente jóvenes de treinta y tantos años". Su estudio concluye que estas personas tienen actividades e intereses asociados a un grupo de edad mucho más joven.

Moloney, Hunt y Evans (2008) analizan la relación entre uso de sustancias e identidad cultural en el discurso de 206 jóvenes asiático-americanos en la cultura rave, mientras que Riley et al. (2008) se centran en el análisis del discurso en relación al consumo de ketamina, profundizando en temas como los derechos y libertades del consumo o la regulación individual.

Anderson y Kavanaugh (2007) y Weir (2000) abordan también aspectos de las raves. Dado que se trata de revisiones, sus trabajos serán comentados en la categoría 5.

\section{Categoría 5. Según estudios de revisión}

En esta categoria han sido incluidas cinco revisiones sobre raves. La mayoría de ellas profundizan fundamentalmente en las drogas, abordando sus efectos y/o consecuencias.

Schwartz y Miller (1997) relacionan los riesgos asociados al uso de MDMA con las particularidades de las raves (largas horas de baile, elevada temperatura, etc.). Entre sus conclusiones destacan que la intoxicación de MDMA puede resultar especialmente peligrosa en estos entornos debido a las altas temperaturas, las largas horas de baile, etc.
Weir (2000) revisa la cultura, las drogas y la prevención de riesgos en las raves, analizando sus valores y significados. Tras analizar el consumo de drogas reflexionan sobre la postura más adecuada ante el consumo; ¿reducción de daños o prohibicionismo?, inclinándose por la primera y proponiendo actuaciones.

Rome (2001) revisa la cultura rave y el uso ilícito de drogas entre los jóvenes, centrándose principalmente en los efectos derivados del uso de sustancias y no profundizando en los aspectos culturales de la fiesta.

Anderson y Kavanaugh (2007) realizan una completa revisión sobre diferentes aspectos de las raves y el consumo de drogas, considerando las implicaciones que se derivan de la postura adoptada al estudiar el fenómeno rave (cultural vs. salud pública). Además, critican la excesiva simplificación de las raves desde la postura de la salud pública, vinculándola a las actuales políticas sobre jóvenes y consumo de drogas.

Por último, Chakraborty, Neogi y Basu (2011) realizan una revisión de las club drugs consumidas en las raves, analizando brevemente la situación de estas fiestas y las club drugs en India.

\section{Categoría 6. Según otros objetivos}

La sexta categoría establecida recoge tres estudios que plantean objetivos diferentes a los recogidos hasta ahora. Van Wijngaart et al. (1999) analizan muestras de orina en tres raves, concluyendo que el método de autoinforme utilizado es un instrumento válido de medida, mientras que Yacoubian y Wish (2006) recogen un total de 96 muestras descubriendo una relación moderada $(K a p p a=0,59)$ entre el autoinforme y el análisis de orina. Lenton y Davidson (1999) analizan el discurso de 83 ravers acerca de las "cosas buenas" o motivaciones para asistir a rave y las que catalogan como "menos buenas". Entre las motivaciones destacan: música, luces y baile (67\%); atmósfera no violenta (31\%); sentimiento de pertenencia comunitario (27\%). Entre las barreras o cosas menos deseables de las raves, el $40 \%$ creía que los ravers "estaban siendo explotados económicamente por los promotores" (Lenton y Davidson, 1999, p. 155).

\section{Discusión}

Las fiestas raves son un contexto recreativo donde el elevado consumo de drogas y las características propias del entorno (elevada temperatura, largas horas de baile...) implican un elevado riesgo para la salud de los participantes. Se trata de un fenómeno fuertemente asentado en distintos países, por lo que su estudio es relevante desde el punto de vista de la salud. En España, Martínez, Pallarés, Espluga, Barruti y Canales (2009) y Martínez, Pallarés, Barruti y Espluga (2011) dejan constancia de la evolución de este fenómeno en Cataluña y Castilla la Mancha. Martínez et al. (2011) afirman que "existen referencias a raves en todo el territorio manchego" (p.26). Pero el mayor exponente de la presencia y evolución de la raves se encuentra en internet, que lo sustenta y fomenta a través de los innumerables foros y webs dedicados a ellas (p. ej., la web www.planetamestizo.org facilita información sobre raves 
organizadas en España, especialmente en su vertiente underground). Por lo tanto, es necesario aportar evidencias empíricas que fundamenten la toma de decisiones y las estrategias de intervención para prevenir y reducir riesgos y daños con este colectivo.

Este trabajo ha realizado una categorización de los estudios sobre raves en función de sus objetivos y resultados. Los resultados han mostrado una escasa producción científica sobre raves. Esto puede deberse a que se trata de un movimiento hermético y poco accesible, especialmente en su forma underground. La revisión sistemática efectuada ha mostrado la necesidad de realizar más estudios empíricos sobre este tipo de fiestas. El hecho de que se celebren en lugares ocultos y/o apartados, y no cuenten con instalaciones de primeros auxilios, puede incrementar el riesgo para los participantes en caso de producirse problemas agudos de salud, lo que debe llevar a una mayor capacitación de estos ravers para manejar y reducir los riesgos que asumen (Fernández-Calderón, 2011d).

La mayoría de estudios analizan el consumo de drogas de los ravers de forma general y no necesariamente vinculado a las fiestas rave. En general, entre las drogas más consumidas por los ravers se encuentran la MDMA, anfetaminas y LSD, tres de las drogas recogidas bajo el término club drugs (Arria et al., 2002) e identificadas como prototípicas de las raves (Anderson y Kavanaugh, 2007). No obstante, el consumo de alcohol, cannabis y cocaína, a pesar de no ser consideradas club drugs, es también muy elevado. Esto debe llevar a replantear si el término se ajusta a las drogas consumidas en las fiestas de baile en general y en las raves en particular.

La escasez de trabajos que han abordado el estudio del policonsumo debe llamar la atención de investigadores y gestores. Se trata de un patrón de uso de drogas normativo en rave, que puede acarrear graves consecuencias para la salud (EMCDDA, 2009; Ricaurte y McCann, 2005) y del que se requiere un estudio en mayor profundidad orientado a la transferencia de conocimientos para reducir sus riesgos (EMCDDA, 2009a). El mayor consumo y policonsumo de drogas detectado por Fernández-Calderón et al. (2011a) podría deberse, en parte, a que se trata del único estudio empírico revisado que analiza este tipo de información en una muestra captada en raves underground.

Esta revisión ha mostrado la necesidad de estudiar el comportamiento de los ravers en relación a la reducción de riesgos y daños. Sólo un trabajo (Hunt et al., 2009a) ha analizado este tipo de información. Conocer qué tipo de estrategias utilizan los ravers y relacionarlas con indicadores de salud debe ser abordardo por la comunidad científica. Igualmente, a pesar de que numerosas organizaciones realizan actividades de reducción de riesgos y daños en este contexto (p.ej., Energy Control, 2012), no se detectan publicaciones que evalúen en qué medida dichas intervenciones/programas son eficaces.

Respecto a los efectos asociados al consumo de drogas en raves, los efectos negativos han sido bien documentados. La mayoría de estudios se han centrado exclusivamente en la MDMA, y no en los de otras drogas o en los efectos negativos del policonsumo. Los resultados de esta revisión son consistentes con lo señalado por Hunt y Evans, (2008) respecto a la escasa atención prestada a los efectos positivos. La importancia de este tipo de efectos para contextualizar el comportamiento de los ravers debe llevar a un mayor estudio de los mismos.

Esta revisión apoya a Anderson y Kavanaugh (2007) respecto a la dominancia de estudios epidemiológicos frente a estudios culturales. Hunt, Moloney y Evans (2009b) plantean que los estudios culturales deben "encontrarse" con los estudios epidemiológicos. Estos autores proponen una aproximación en la que las fortalezas teóricas y metodológicas de la postura cultural sean utilizadas desde una perspectiva que nos permita comprender el rol de las drogas, los placeres y los riesgos. Una apropiada contextualización de los riesgos asociados a las raves debe considerar no sólo las drogas utilizadas (con sus efectos y riesgos), sino también la persona que las consume (con sus experiencias, creencias, motivaciones...) y el contexto en que son consumidas (cultura, tipo de fiesta...). Como afirma Romani (2008) "para entender la variabilidad de efectos de las drogas no podemos disociar la sustancia consumida del sujeto consumidor ni del contexto donde se da el acto de consumo" (p. 302).

La presente revisión presenta algunas limitaciones. La decisión de incluir sólo estudios en inglés y castellano podría afectar a la generalización de las conclusiones obtenidas. Además, la no inclusión de otras tipologías documentales (p.ej., tesis, comunicaciones a congresos...) podría haber supuesto que algunas investigaciones sobre raves no fueran revisadas.

A pesar de las limitaciones, se ha aportado información de utilidad en varios sentidos. Por una parte, se ha enmarcado la investigación sobre raves en seis categorías. Por otra, los resultados y conclusiones obtenidos pueden servir de base para el desarrollo de actuaciones preventivas y de reducción de riesgos/daños. Además, a partir de los resultados obtenidos, se han puesto de manifiesto necesidades de investigación/ conocimiento sobre las raves que pueden resultar orientativas en el diseño y realización de futuros estudios y actuaciones preventivas.

\section{Reconocimientos}

Este trabajo se ha desarrollado en el marco del proyecto de investigación con referencia PI10/01155 financiado por el Instituto de Salud Carlos III y fondos FEDER.

\section{Conflicto de intereses}

No existe conflicto de intereses

\section{Referencias}

Adlaf, E. M. y Smart, R. G. (1997). Party subculture or dens of doom? An epidemiological study of rave attendance and drug use patterns among adolescent students. Journal of Psychoactive Drugs, 29, 193-198. 
Anderson, T. L. y Kavanaugh, P. R. (2007). A "Rave" review. Conceptual interests and analytical shifts in research on rave culture. Sociology Compass, 1, 499-519. doi: 10.1111/j.1751-9020.2007.00034.x

Arria, A. M., Yacoubian, G. S., Fost, E. y Wish, E. D. (2002). Ecstasy use among club rave attendees. Archives of Pediatric Adolescent Medicine, 156, 295-296. doi: doi:10.1001/archpedi.156.3.295

Barrett, S. P., Gross, S. R., Garand, I. y Pihl, R. O. (2005). Patterns of simultaneous polysubstance use in Canadian rave attendees. Substance Use and Misuse, 40, 15251537. doi: 10.1081/ JA-200066866

Barrett, S. P., Darredeu, C. y Pihl, R. O. (2006). Patterns of simultaneous polysubstance use in drug using university students. Human Psychopharmacology, 21, 255-263. doi: 10.1002/hup.766

Bennett, A. (1999). Subcultures or neo-tribes? Rethinking the relationship between style and musical taste. Sociology, 33, 599617. doi: $10.1177 /$ S0038038599000371

Bennett, A. (2001). Cultures of popular music. Philadelphia: Open University Press.

Boeri, M. W., Sterk, C. E. y Elifson, K. W. (2004). Rolling beyond raves: Ecstasy use outside the rave setting. Journal of Drug Issues, 34, 831-860. doi: 10.1177/002204260403400406

Boys, A., Lenton, S. y Norcross, K. (1997). Polydrug use at raves by a Western Australian sample. Drug and Alcohol Review, 16, 227-234. doi: $10.1080 / 09595239800187411$

Chakraborty, K., Neogi, R. y Basu, D. (2011). Club drugs: Review of the 'rave' with a note of concern for the Indian scenario. Indian Journal of Medical Research, 133, 594-604.

Collin, M. (2002). Estado Alterado. La Historia de la cultura del éxtasis y del acid house. Barcelona: Alba Editorial.

Engels, R. C. M. E. y Ter Bogt, T. (2004). Outcome expectancies and ecstasy use in visitors of rave parties in the Netherlands. European Addiction Research, 10, 156-162. doi: 10.1159/000079836

European Monitoring Centre for Drugs and Drug Addiction (2009). Polydrug use: Pattens and responses. Publications Office of the European Union, Luxembourg. Obtenido 03/02/2012 de http://www. emcdda.europa.eu/attachements.cfm/att_93217_EN_EMCDDA_ SI09_polydrug\%20use.pdf

European Monitoring Centre for Drugs and Drug Addiction (2011). Annual Report on the state on the drugs problem in Europe, Publications Office of the European Union, Luxembourg. Obtenido 26/02/2012 de http://www.emcdda.europa.eu/attachements.cfm/ att_143743_EN_EMCDDA_AR2011_EN.pdf

Fernández-Calderón, F., Lozano, O. M., Vidal, C., Gutiérrez, J., Vergara, E. y González-Saiz, F. (2011a). Polysubstance use patterns in underground rave attenders: A cluster analysis. Journal of Drug Education, 41, 183-202. doi: 10.2190/DE.41.2.d

Fernández-Calderón, F., Lozano-Rojas, O. M., Bilbao-Acedos I., RojasTejada, A. J., Vidal-Giné, C., Vergara-Moragues, E. y González-Saiz, F. (2011b). Análisis en el perfil y patrón de consumo de drogas de hombres y mujeres que asisten a fiestas rave. Trastornos Adictivos, $12,167-74$.
Fernández-Calderón, F., Lozano-Rojas, O. M., Bilbao-Acedos I., RojasTejada, A. J., Vidal-Giné, C., Vergara-Moragues, E. y González-Saiz, F. (2012). Efectos asociados al policonsumo de drogas en fiestas rave. Salud y Drogas, 12, 37-59.

Fernández-Calderón, F. (2011d). What happens at underground rave? Risk, research and harm reduction. Journal of Socialomics, 1 :e102. doi:10.4172/jsc.1000e102.

Forsyth, A. J. M. (1996). Places and patterns of drug use in the Scotish dance scene. Addiction, 9, 511-521.

Goulding, C., Shankar, A. y Elliott, R. (2002). Working weeks, rave weekends: Identity fragmentation and the emergence of new communities. Consumption Markets and Culture, 5, 261-284. doi: 10.1080/1025386022000001406

Goulding, C. y Shankar, A. (2004). Age is Just a Number: Rave culture and the cognitively young "thirty something." European Journal of Marketing, 38, 641-658. doi: 10.1108/03090560410529268

Gross, S. R., Barrett, S. P., Shestowsky, J. S. y Pihl, R. O. (2002). Ecstasy and drug consumption patterns: A Canadian rave population study. Canadian Journal of Psychiatry, 47, 546-551.

Hill, A. (2002). Acid House and Thatcherism: noise, the mob, and the English countryside. British Journal of Sociology, 53, 89-105.

Hunt, G. y Evans, K. (2008). "The great unmentionable": Exploring the pleasures and benefits of ecstasy from the perspectives of drug users. Drugs: Education, Prevention \& Policy, 15, 329-349. doi: 10.1080/09687630701726841

Hunt, G., Evans, K., Moloney, M. y Bailey., N. (2009a). Combining different substances in the dance scene: enhancing pleasure, managing risk and timing effects. Journal of drug issues, 39, 495-522.

Hunt, G., Moloney, M. y Evans, K. (2009b): Epidemiology meets cultural studies: studying and understanding youth cultures, clubs and drugs. Addiction Research and Theory, 17, 601-621.

Kavanaugh, P. R. y Anderson, T. L. (2008). Solidarity and drug use in the electronic dance music scene. The Sociological Quarterly, 49, 181208. doi: 10.1111/j.1533-8525.2007.00111.x

Krul, J. y Girbes, A. R. (2011a). Gamma-hydroxybutyrate: Experience of 9 years of gamma-hydroxybutyrate (GHB)-related incidents during rave parties in the Netherlands. Clinical Toxicology, 49, 311-315. doi: 10.3109/15563650.2011.576253

Krul, J., Blankers, M. y Girbes, A. R. (2011b). Substance-related Health problems during rave parties in the Netherlands (1997-2008). Plos One, 6, e29620. doi:10.1371/journal.pone.0029620

Lenton, S., Boys, A. y Norcross, K. (1997). Raves, drugs and experience: Drug use by a sample of people who attend raves in Western Australia. Addiction, 92, 1327-1337.

Lenton, S. y Davidson, P. (1999). Raves, drugs, dealing and driving: Qualitative data from a West Australian sample. Drug and Alcohol Review, 18, 153-161. doi: 10.1080/09595239996581

Martínez, D. P., Pallarés, J., Espluga, J., Barruti, M., y Canales, G. (2009). Observatorio de nuevos consumos de drogas en el ámbito juvenil. Informe 2008, Fundación IGENUS. Obtenido 26/11/2012 en http://www.fund-igenus.org/esp/ investigacio/pdf/ InformeObservatori2008_esp.pdf 
Martínez, D.P., Pallarés, J., Barruti, M., y Espluga, J. (2011). Sistema de Información Continua Sobre el Consumo de Drogas entre los jóvenes en Castilla-La Mancha (SICCAM). Informe 2010. Obtenido 03/03/2013 de http://www.od.jccm.es/admin/modulos/ publicaciones/pdf/123_28703196.pdf

McCaughan, J. A., Carlson, R. G., Falck, R. S. y Siegal, H. A. (2005). From "Candy Kids" to "Chemi-Kids": A typology of young adults who attend raves in the Midwestern United States. Substance Use and Misuse, 40, 1503-23. doi: 10.1081/JA-200066830

Moloney, M., Hunt, G. y Evans, K. (2008). Asian American identity and drug consumption: From acculturation to normalization. Journal Ethnic Substance Abuse, 7 (4), 376-403.

Observatorio Español sobre Drogas (2007). Encuesta domiciliaria sobre alcohol y drogas en España (EDADES), 1995-2007. Madrid: Delegación del Gobierno para el Plan Nacional sobre Drogas, Ministerio del Interior.

Reynolds, S. (1999). Generation ecstasy: Into the world of techno and rave culture. New York: Routledge.

Ricaurte, G. A. y McCann, U. D. (2005). Recognition and management of complications of new recreational drug use. The Lancet, 365 (9477), 2137-2145.

Riley, S. C. E., James, C., Gregory, D., Dingle, H. y Cadger, M. (2001). Patterns of recreational drug use at dance events in Edinburgh, Scotland. Addiction, 96, 1035-1047. doi: 10.1080/09652140120053093

Riley, S. C. E. y Hayward, E. (2004). Patterns, trends, and meanings of drug use by dance-drug users in Edinburgh, Scotland. Drugs: Education, Prevention and Policy, 11, 243-262. doi: 10.1080/09687630310001643111

Riley, S., Morey, Y. y Griffin, C. (2008). Ketamine: The divise dissociative. A discourse analysis of the constructions of ketamine by participants of a free party (rave) scene. Adiction Research and Theory, 16, 217 230. doi: $10.1080 / 16066350801983715$

Romani, 0. (2008). Políticas de drogas: prevención, participación y reducción del daño. Salud Colectiva, 4 (3), 301-318.

Rome, E. S. (2001). It's a rave new world: Rave culture and illicit drug use in the young. Cleveland Clinic Journal of Medicine, 68, 541 550.

Sanders, B. (2006) Young People, Clubs and Drugs. En B. Sanders (Ed.). Drugs, clubs and young people, (pp. 1-12). Hampshire, Ashgate.

Schwartz, R. H. y Miller, N. S. (1997). MDMA (Ecstasy) and the rave: A review. Pediatrics, 100, 705-708.

Ter Bogt, T. F. M. y Engels, R. C. M. E. (2005). "Partying" hard: Party style, motives for and effects of MDMA use at rave parties. Substance Use and Misuse, 40, 1479-1502. doi: 10.1081/JA-200066822

Tossmann, P., Boldt, S. y Tensil, M. D. (2001). The use of drugs within the techno party scene in European metropolitan cities. European Addiction Research, 7, 2-23. doi: 10.1159/000050709

Van Sassenbroeck, D. K., Calle, P. A., Rousseau, F. M., Verstraete, A. G., Belpaire, F. M., Monsieurs, K. G. et al. (2003). Medical problems related to recreational drug use at nocturnal dance parties. European Journal of Emergency Medicine, 10, 302-308.
Van Wijngaart, V., Braam, R., Bruin, D., Fris, M., Maalsté, N. J. M. y Verbraeck, H. T. (1999). Ecstasy use at large-scale dance events in the Netherlands. Journal of Drug Issues, 29, 679-702.

Weir, E. (2000). Raves: A review of the culture, the drugs and the prevention of harm. Canadian Medical Association Journal, 162, 1843-1848.

Yacoubian, G. S., Boyle, C., Harding, C. A. y Loftus, E. A. (2003). It's a rave new world: Estimating the prevalence and perceived harm of ecstasy and other drug use among club rave attendees. Journal of Drug Education, 33, 187-196.

Yacoubian, G. S., Miller, S., Pianim, S., Kunz, M., Orrick, E., Link, T., et al. (2004). Toward and ecstasy and other club drug (EOCD) prevention intervention for rave attendees. Journal of Drug Education, 34, 41-59.

Yacoubian, G. S. y Wish, E. D. (2006). Exploring the validity of selfreported ecstasy use among club rave attendees. Journal of Psychoactive Drugs, 38, 31-34.

Yacoubian, G. S. y Peters, R. J. (2007). An exploration of recent club drug use among rave attendees. Journal of Drug Education, 37, 145-161. 